\title{
GENIE Production Release 2.10.0
}

M. Alam ${ }^{1}$, C. Andreopoulos ${ }^{2,3}$, M. Athar ${ }^{1}$, A. Bodek ${ }^{4}$, E. Christy ${ }^{5}$, B. Coopersmith ${ }^{4}$, S. Dennis ${ }^{6}$, S. Dytman ${ }^{7}$, H. Gallagher ${ }^{8}$, N. Geary ${ }^{7}$, T. Golan ${ }^{4,9}$, R. Hatcher ${ }^{9}$, K. Hoshina ${ }^{10}$, J. Liu ${ }^{11}$, K. Mahn ${ }^{12}$, C. Marshall ${ }^{4}$, J. Morrison ${ }^{12}$, M. Nirkko ${ }^{13}$, J. Nowak ${ }^{14}$, G. N. Perdue ${ }^{9}$, and J. Yarba ${ }^{9}$

${ }^{1}$ Department of Physics, Aligarh Muslim University, Aligarh-202 002, India

${ }^{2}$ Department of Physics, University of Liverpool, Liverpool, L69 7ZE, UK

${ }^{3}$ Particle Physics Department, STFC Rutherford Appleton Laboratory, Harwell Oxford, OX11 0QX, UK

${ }^{4}$ Department of Physics, University of Rochester, Rochester, New York 14610 USA

${ }^{5}$ Dept. of Physics, Hampton University, Hampton, VA 23668, USA

${ }^{6}$ Department of Physics, University of Warwick, Gibbet Hill Road, Coventry CV4 7AL, UK

${ }^{7}$ Department of Physics and Astronomy, University of Pittsburgh, Pittsburgh, Pennsylvania 15260, USA

${ }^{8}$ Department of Physics and Astronomy, Tufts University, Medford, Massachusetts 02155, USA

${ }^{9}$ Fermi National Accelerator Laboratory, Batavia, Illinois 60510, USA

${ }^{10}$ Dept. of Physics and Wisconsin IceCube Particle Astrophysics Center, University of

Wisconsin, Madison, Wisconsin 53706, USA

${ }^{11}$ Department of Physics, College of William \& Mary, Williamsburg, Virginia 23187, USA

${ }^{12}$ Department of Physics and Astronomy, Michigan State University, East Lansing, MI 48824, USA

${ }^{13}$ University of Bern, Albert Einstein Center for Fundamental Physics, Laboratory for High Energy Physics (LHEP), Bern, Switzerland

${ }^{14}$ Department of Physics, Lancaster University, Lancaster, LA0 4YW, United Kingdom

February 9, 2020

\begin{abstract}
GENIE (Generates Events for Neutrino Interaction Experiments) is a neutrino Monte Carlo event generator that simulates the primary interaction of a neutrino with a nuclear target, along with the subsequent propagation of the reaction products through the nuclear medium. It additionally contains libraries for fully-featured detector geometries and for managing various types of neutrino flux. This note details recent updates to GENIE, in particular changes introduced into the newest production release, version 2.10.0.
\end{abstract}




\section{Introduction}

GENIE [1] is a neutrino event generator created to be the "universal event generator" discussed during the NUINT conference series. It was designed to provide an accessible, extensible framework with many convenience tools leveraging existing HEP software like PYTHIA [2], ROOT [3], and LHAPDF [4].

This note describes the changes in the GENIE generator code base between production release version 2.8.0 (and its subsequent patches) and the new production release, 2.10.0. The GENIE code is available through a publicly visible Subversion repository or via a source tar-file. Details for both methods of accessing the code are available on the GENIE homepage at http://genie. hepforge.org.

GENIE 2.10.0 is a "model introduction release." Broadly speaking, the GENIE collaboration releases two kinds of updates for the generator code - model introduction and physics tuning releases. Model introduction releases aim to incorporate new models into the code base but they do not incorporate them into the default global physics model. Physics tuning releases modify the default global physics model. Note that these categories are not hard and fast rules - occasionally the global physics model will perform slightly differently after a bug is removed from the code in a model introduction release, and we will occasionally introduce new models in physics tuning releases.

\section{Modifications to Existing Models}

In this section we describe new models that can be utilized as options within the existing GENIE generators. None of these are turned on by default (with the exception of the inclusion of the W/Z propagator terms in the DIS cross section), but can be enabled by the user in the UserPhysicsOptions.xml file.

\subsection{Nuclear Models}

Quasi-elastic (QE) models based on Llewelyn Smith [6] and the Fermi Gas Model [7] are presently the standard in neutrino event generators because of their simplicity and wide applicability. They qualitatively describe inclusive electron scattering data, but struggle to explain exclusive electron and neutrino cross section results consistently over a wide range of energies and kinematics 8 12. One explanation for this is an incomplete description of nuclear effects, with nucleon-nucleon correllations such as Meson Exchange Currents expected to play a large role [13 19]. These modify both the nucleon momentum distribution and the shape of the cross section. Two models were added to GENIE to capture these effects: a Transverse Enhancement model (TEM) [20] and an Effective Spectral Function model [5].

\subsubsection{Transverse Enhancement}

In the TEM, nuclear effects like those expected from Meson Exchange Currents are modeled as $Q^{2}$ dependent modifications to the elastic proton and neutron magnetic form factors. The exact $Q^{2}$ dependence is extracted from fits of the transverse quasi-elastic response function from electron 

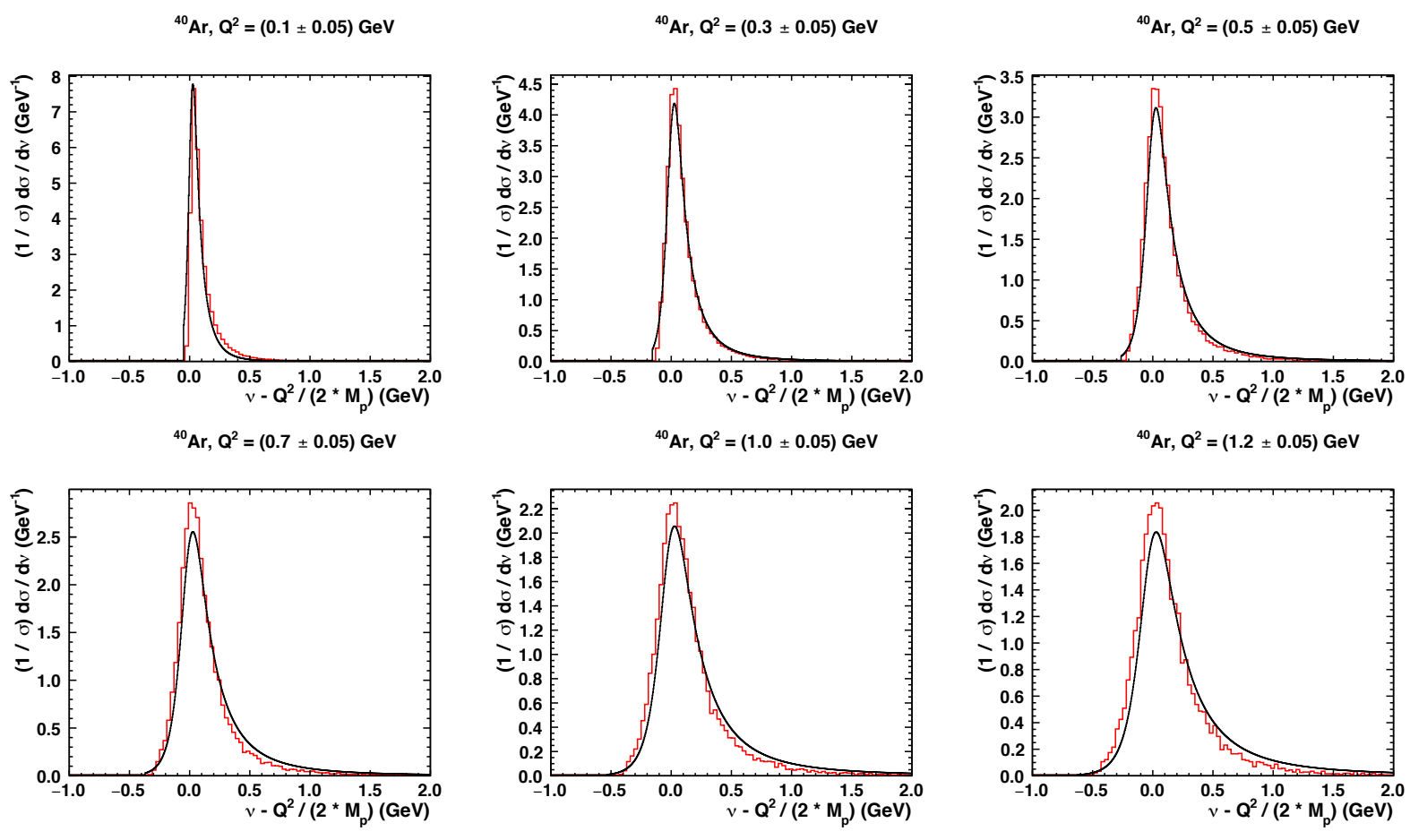

${ }^{40} \mathrm{Ar}, \mathrm{Q}^{2}=(1.5 \pm 0.05) \mathrm{GeV}$

${ }^{40} \mathrm{Ar}, \mathrm{Q}^{2}=(2.0 \pm 0.05) \mathrm{GeV}$
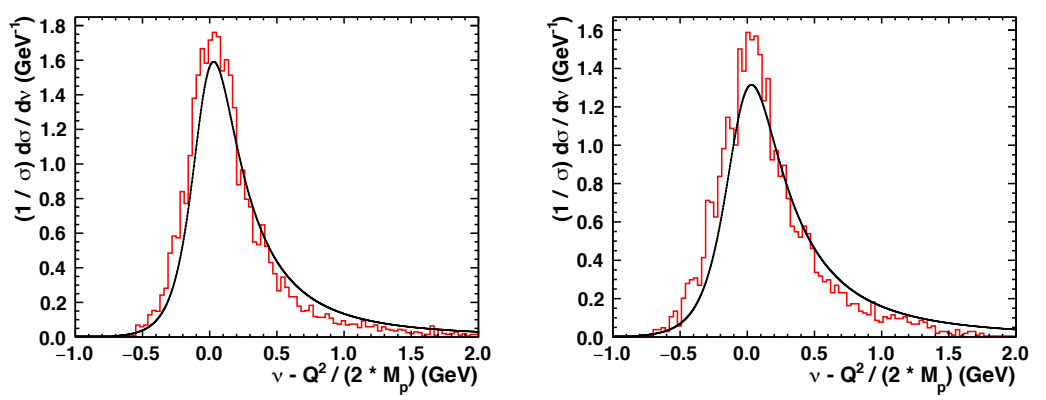

Figure 1: Comparisons between the $\psi^{\prime}$ superscaling parameter as a function of $\nu-Q^{2} /\left(2 M_{p}\right)$, where $\nu$ is the energy transfer to the hadronic system during the neutrino scattering event, $Q^{2}$ is the four momentum transfer from the neutrino, $M_{p}$ is the target nucleon mass. The curve extracted from electron scattering data [5] (the smooth black curve) is compared to the computation produced by GENIE (the red histogram) for different values of $Q^{2}$ on Argon-40. 
Neutrino Cross Section $-v+\mathbf{n} \rightarrow \mathbf{p}+\mu^{-}$

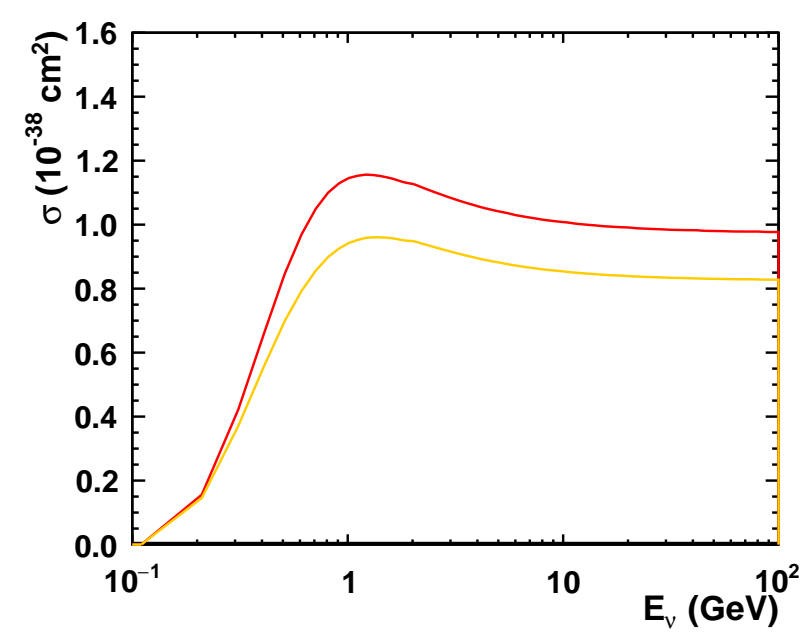

Figure 2: The charged-current quasielastic cross section for neutrinos with the default Llewelyn Smith model in orange and the new Effective Spectral Function model in red. The cross section is computed on Carbon and then scaled by the number of neutrons.

scattering data. This model by itself doesn't emit nucleons. However, 1 or 2 nucleons are emitted through the effective spectral function.

\subsubsection{Effective Spectral Functions}

Nuclear models such as the Local Thomas Fermi Gas [21, global Fermi Gas [7, 22, 23], or BenharFantoni Spectral Function 24,25 provide different momentum distributions which changes the shape of the quasielastic cross section. Final state interactions at the Feynman diagram level change the shape of the differential cross section in energy transfer, with an increase of strength in the tail of the distribution and an decrease in the peak. These interactions are included in superscaling calculations 26. The Effective Spectral Function model in GENIE 2.10.0 is fitted to these model's predictions of $1 / \sigma \times d \sigma / d \nu$, where $\nu$ is the energy transfer to the hadronic system during the scattering event, at various values of $Q^{2}$, where $Q^{2}$ is the four momentum transfer from the neutrino. See Figure 1 for a comparison of the superscaling model prediction to the predictions of implementation in GENIE.

The TE Model uses a modified transverse form factor to add to the strength to higher energy loss. Used with the Effective Spectral Function as the nuclear model, the TEM effectively provides a Meson Exchange Currents. Together, they give a complete prescription that is in agreement with a wide range of electron scattering data. The EFS and TEM models may be activated independently if the user chooses and they are not active by default in GENIE 2.10.0. Figures 2 and 3 show the neutrino and antineutrino cross sections on Carbon as a function of energy with the EFS active (but TEM disabled) scaled by the number of protons or neutrons as appropriate. 
Anti-Neutrino Cross Section $-\bar{\nabla}+\mathbf{p} \rightarrow \mathbf{n}+\mu^{+}$

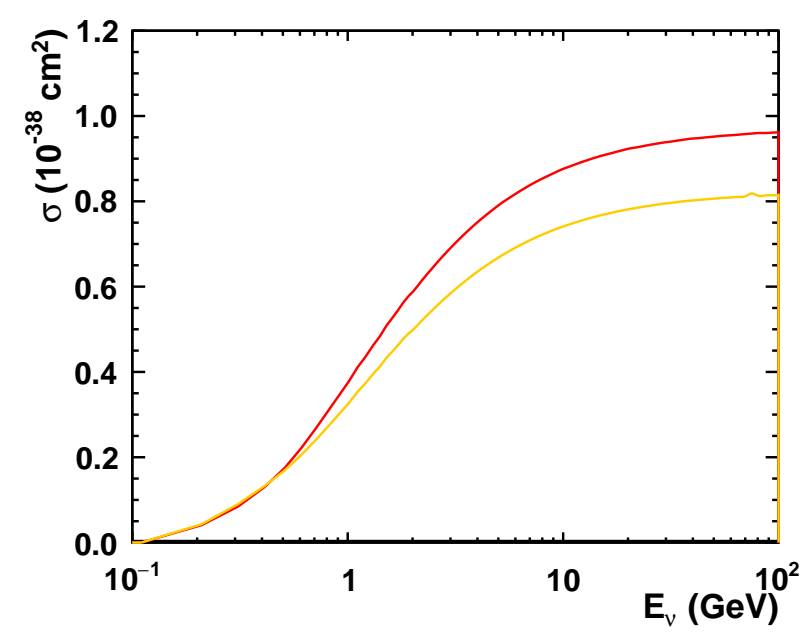

Figure 3: The charged-current quasielastic cross section for antineutrinos with the default Llewelyn Smith model in orange and the new Effective Spectral Function model in red. The cross section is computed on Carbon and then scaled by the number of protons.

\subsubsection{Activating Effective Spectral Functions and Transverse Enhancement}

By default, GENIE uses the Relativistic Fermi Gas with the Bodek-Ritchie modifications [27, 28 and no Transverse Enhancement. To change the model used, make edits to the configuration file found in UserPhysicsOptions.xml. Activate Transverse Enhancement by changing the parameter UseElFFTransverseEnhancement to true. Similarly, the Effective Spectral Functions is activated by changing the NuclearModel to genie: :EffectiveSF/Default. To activate both models simultaneously, make both of the changes described above.

\subsection{Hadronization Models}

Eta mesons, like $\pi^{0} \mathrm{~s}$, have purely electromagnetic decays into photons and can therefore mimic electron neutrino appearance. For this reason, their simulation is important for oscillation experiments. Prior to this GENIE release, $\eta$ mesons were produced through two mechanisms, the decay of baryon resonances, and PYTHIA fragmentation. The result is a kinematic gap over which $\eta$ mesons are not produced - non-resonant inelastic events with invariant masses too low to be fragmented by PYTHIA. These events are handled by the KNO-based part of the AGKY model [29]. In this model, mesons are produced in pairs with a net charge of zero, according to probabilities assigned via the KNO-Prob* values in UserPhysicsOptions.xml. Two new values have been added in this release, KNO-ProbPiOEta and KNO-ProbEtaEta. The ability to create $\eta$ mesons over all values of $\mathrm{W}$ makes possible background studies for oscillation experiments. Both are currently set to zero in 2.10.0, but we expect that they will be tuned to non-zero values in the next GENIE physics release. Figure 4 shows the effect of setting these parameters to a value of 0.1 , with a corresponding decrease in other KNO-Prob values. These are unrealistically large values, and are used here for the purposes of illustrating the kinematic range that is being affected by these parameters. 


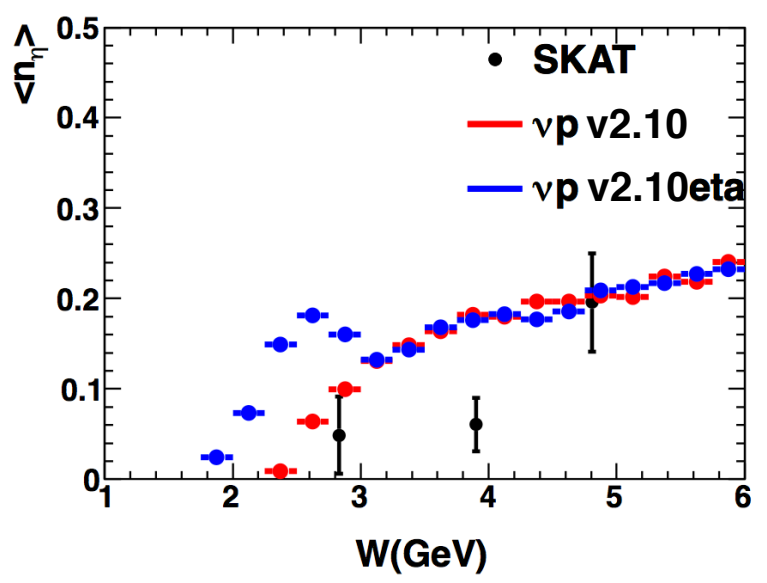

Figure 4: Eta production rate measurements from the SKAT experiment 30, compared with the GENIE default prediction (red) and the GENIE prediction with eta production parameters set to large non-zero values (blue).

\subsection{Intranuclear Rescattering Models}

This release includes a new determination of the relative probabilities of pion interaction fates in the hA intranuclear cascade simulation. The default code for choice of final state channel uses data and model results, e.g. charge exchange vs. absorption, for a Fe target for all probes. These results are then unchanged for other nuclei which produces $\sim 20 \%$ deviations from pion interaction data, which is much more available than for protons or neutrons. The new alternate hA2014 model includes a wide range of data for other nuclei for $\pi^{ \pm}$so that much less extrapolation is needed. To enable it, set the parameter HadronTransp-Model to genie: :hAIntranuke2014/Default where the default value is genie: :hAIntranuke/Default. The new data is mostly from Ashery ( $\mathrm{Li}, \mathrm{C}$, $\mathrm{Al}, \mathrm{Fe}, \mathrm{Nb}, \mathrm{Bi}$ ) 31 but includes other sources $32-36$. To calculate the fractions for hAIntranuke, total cross sections [37] and additional inputs are also needed. For higher energy pions (Ashery highest energy is $315 \mathrm{MeV}$ ), the Mashnik CEM03 Monte Carlo calculations for Fe [38, 39] are still used. Other nuclei are simulated assuming $A^{\frac{2}{3}}$ scaling which is a good approximation when there is no data to use directly. At low energies, 0 and $50 \mathrm{MeV}$, the required values can be constructed from existing data $32,40,41]$. Here, the $\pi^{-}$total cross sections should be larger than those for $\pi^{+}$. Splines are built with almost all available data. Some data values cause sharp features in the splines. In those cases, individual data points were shifted within the estimated error to produce a smooth result. Total inelastic cross section data from Ashery at $85 \mathrm{MeV}$ is incompatible with the newer Aniol 35] data and was therefore not used. Total absorption cross section data from Nakai [42] is not compatible with Ashery [31] and was not used. New results for $\pi^{+} C$ are shown in Figure 5 for the total absorption and inelastic cross sections.

\subsection{Cross Section Models}

In 2.10.0 we made one significant change that impacts the global physics model - the GNU Scientific Library [43] is the new default numerical integrator. This change caused some minor numerical 

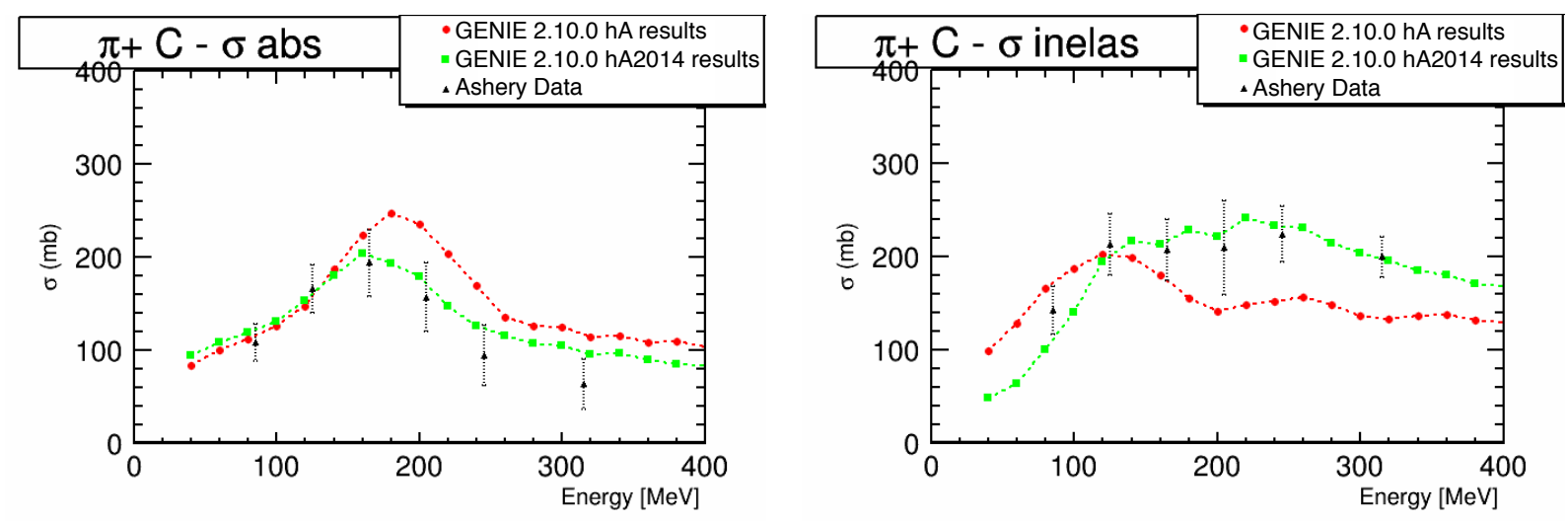

Figure 5: Comparison of new total cross sections for $\pi^{+} C$ for new $h A 2014$ model with default model $h A$.

perturbations in the behavior of many of the cross section calculations. A sign error for the $\Delta_{s}$ contribution to the axial form factor in neutrino-neutron $\mathrm{NC}$ elastic scattering has also been fixed, resulting in a roughly $25 \%$ decrease in the cross section for this process.

\subsubsection{High Energy Cross Sections}

In order to improve the accuracy of GENIE DIS calculations at energies greater than $100 \mathrm{GeV}$, the propagator terms for the $\mathrm{W}$ and $\mathrm{Z}$ bosons have been added to the DIS CC and NC differential cross section calculations. Since this is clearly an improvement to the calculation, and since it has negligible impact on the $\mathrm{CC}$ cross section below $50 \mathrm{GeV}$, it is included in the default cross section calculation.

\subsubsection{Berger-Sehgal Resonant Pion}

The Berger-Sehgal (BS) [44 and Kuzmin-Lyubushkin-Naumov (KLN) 45 models for $N^{*}$ resonances are very similar to the default Rein-Sehgal [46] model, but include the effects due to the muon mass. BS includes an extra diagram that is not found in KLN. Much of the original code for the resonance couplings is untouched. The new models are enabled by changing the resonance (RES) model in UserPhysicsOptions.xml from genie: :ReinSehgalRESPXSec/Default (default Rein-Sehgal model) to either genie: :BergerSehgalRESPXSec2014/Default (for BS) or genie: :KuzminLyubushkinNaumovRESPXSec2014/Default (for KLN).

Work in MiniBooNE collaboration also improved the form factors which have remained unchanged in the Rein-Sehgal resonance model [47, 48]. These are also included with parameters (minibooneGV and minibooneGA for new vector and axial form factors) in UserPhysicsOptions . xml.

GENIE validations are shown in Figure 6 for $1 \mathrm{GeV} \nu_{\mu} C \mathrm{CC}$ interactions. Distributions for true $Q^{2}$ and $\pi^{+}$kinetic energy are supplied. Including the muon mass has the largest effect close to threshold. The differences in the plots are mostly due to changes in form factors.

Figures 7 and 8 show the total charged-current cross section / energy for muon neutrinos and antineutrinos. Careful inspection of the total cross section shows some small changes resulting from the switch to gsl and the inclusion of the $\mathrm{W} / \mathrm{Z}$ propagator terms, however, differences between the 

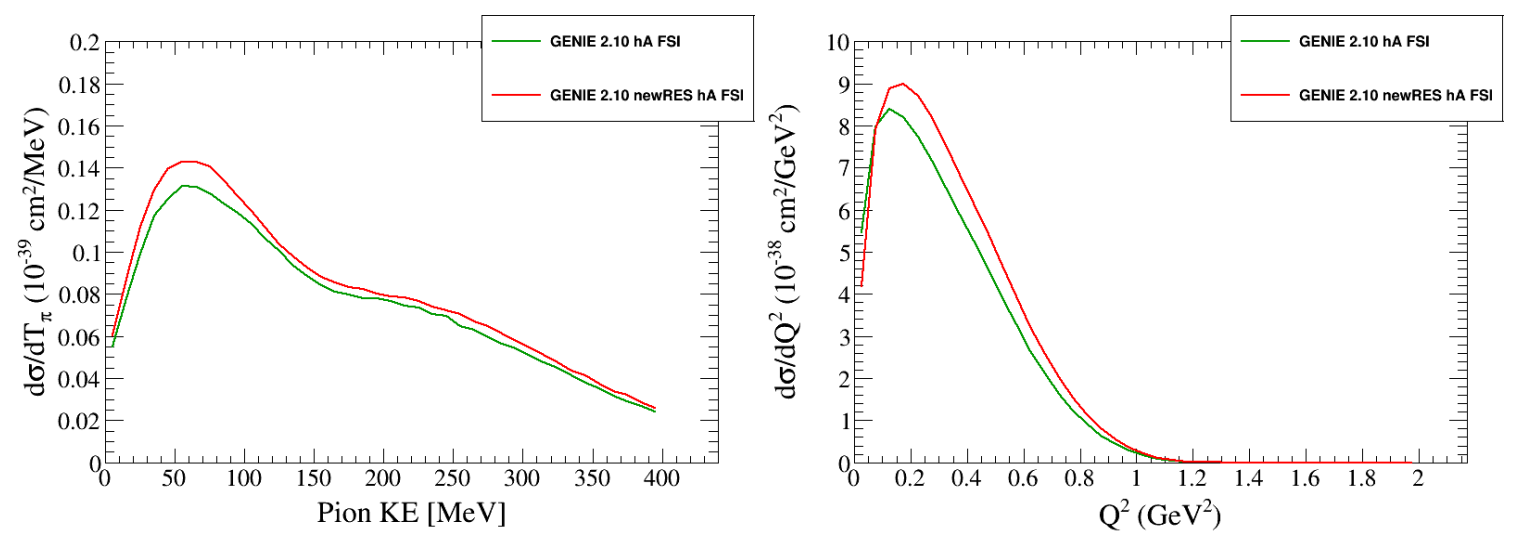

Figure 6: Comparison of new model (Berger-Sehgal with new form factors) with default model.

2.10 and 2.8.6 cross sections are generally less than $1 \%$ for neutrino energies less than $100 \mathrm{GeV}$.

\section{New Interaction Models: Single Kaon Production}

There is a model for one entirely new interaction process in this release, neutrino-production of single kaons from Athar et al. [49], generating events in the channels $\nu_{l}+p \rightarrow l^{-}+K^{+}+p$, $\nu_{l}+n \rightarrow l^{-}+K^{0}+p$, and $\nu_{l}+n \rightarrow l^{-}+K^{+}+n$. This is the first $\Delta S=1$ process to be included in GENIE. When running on a nuclear target, the process is embedded in the default nuclear model (for simulation of Fermi motion and intranuclear rescattering), in the same way that the free nucleon QEL model is incorporated into the nuclear model. The cross section on a nucleus is taken as $\mathrm{Z}(\mathrm{N})$ times the free proton (neutron) cross section.

\subsection{Single Kaon Production}

Figure 9 shows the differential distributions produced in the scattering of $1.5 \mathrm{GeV}$ muon neutrinos in the channel $\nu_{\mu}+p \rightarrow \mu^{-}+K^{+}+p$, as calculated using a standalone $\mathrm{C}++$ code that was validated against both the original Fortran calculation, and the 2.10 GENIE implementation. In GENIE, this is implemented as a 4-fold differential cross section calculation in the outgoing lepton energy and scattering angle, the kaon energy, and $\phi_{K q}$ (defined as the angle between the kaon- $\vec{q}$ plane and the lepton plane). Handling integration of 4-fold differential cross sections with the necessary precision was one of the technical drivers of the transition to GSL that was part of this release. The range of validity of this model is for neutrino energies up to $2 \mathrm{GeV}$ (although the model will run for energies above that), and no attempt has been made in this release to re-tune other processes which produce kaons, such as associated production.

This model is included as a specific implementation (in the GENIE code base as AlamSimoAtharVacasSKPXSec2014) of single-kaon production processes, which are identified by a new enum value (kScSingleKaon) in the ScatteringType object and related classes. The DIS-CC-SINGLEK event generator is currently configured to use this model as its default, and selecting the SingleKaon event generator list (i.e. as an input to gmkspl or gevgen), will enable this as the sole event generation thread. 

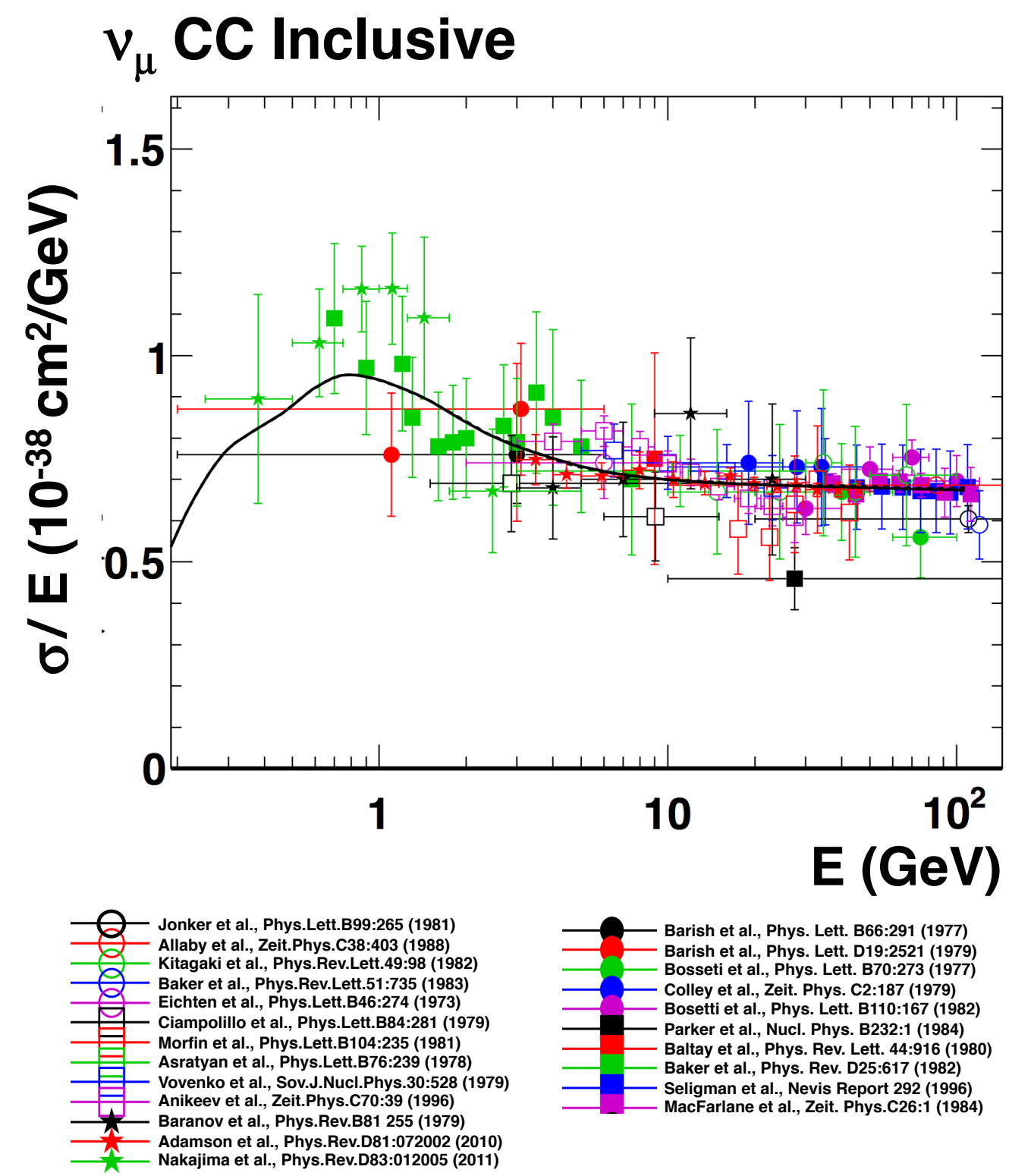

Figure 7: The GENIE 2.10.0 (solid black line), and 2.8.6 (dashed black line) muon neutrino inclusive CC cross section compared to data. 


\section{$\overline{v_{\mu}}$ CC inclusive}
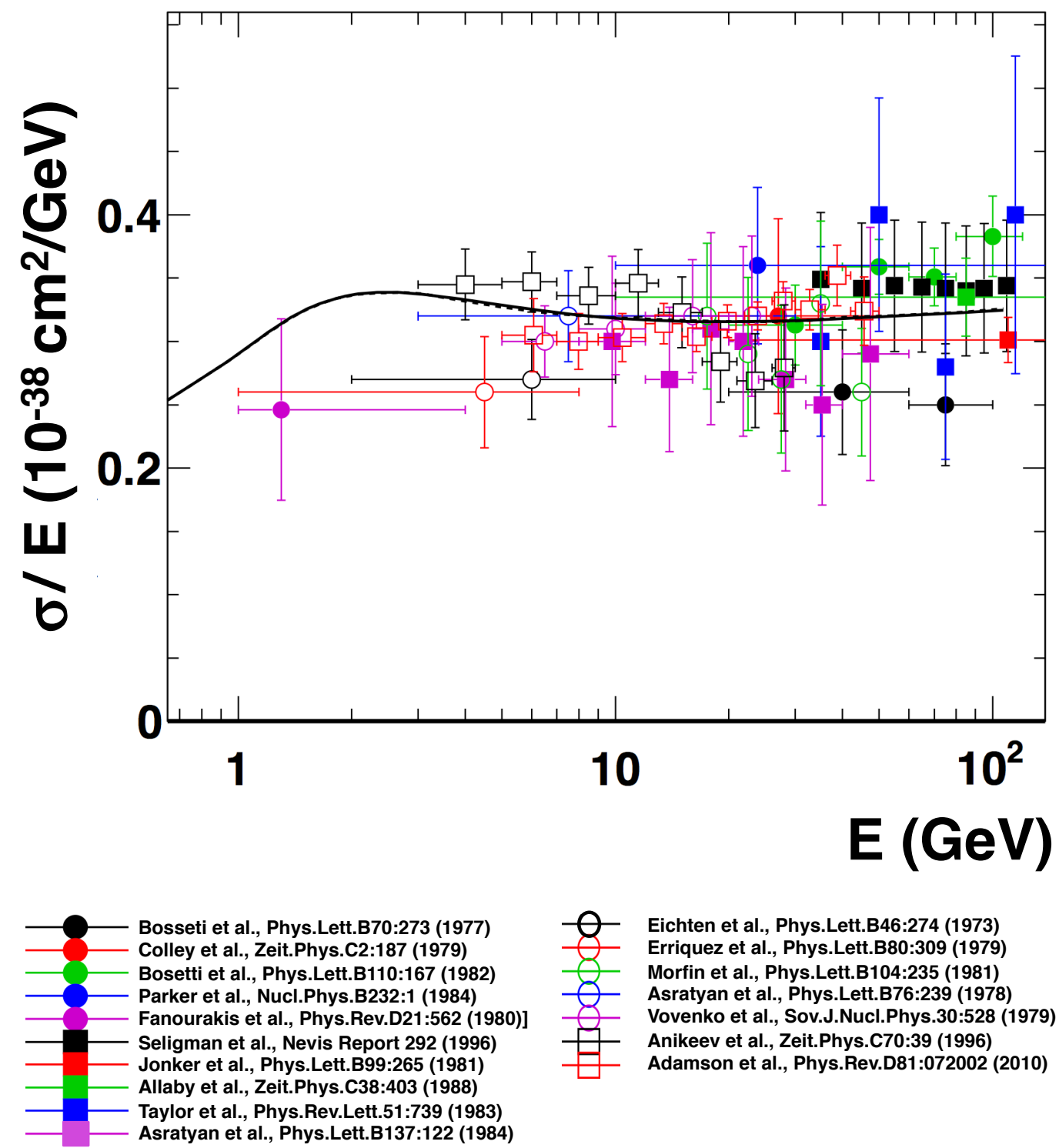

Figure 8: The GENIE 2.10.0 (solid black line), and 2.8.6 (dashed black line) muon anti-neutrino inclusive $\mathrm{CC}$ cross section compared to data. 

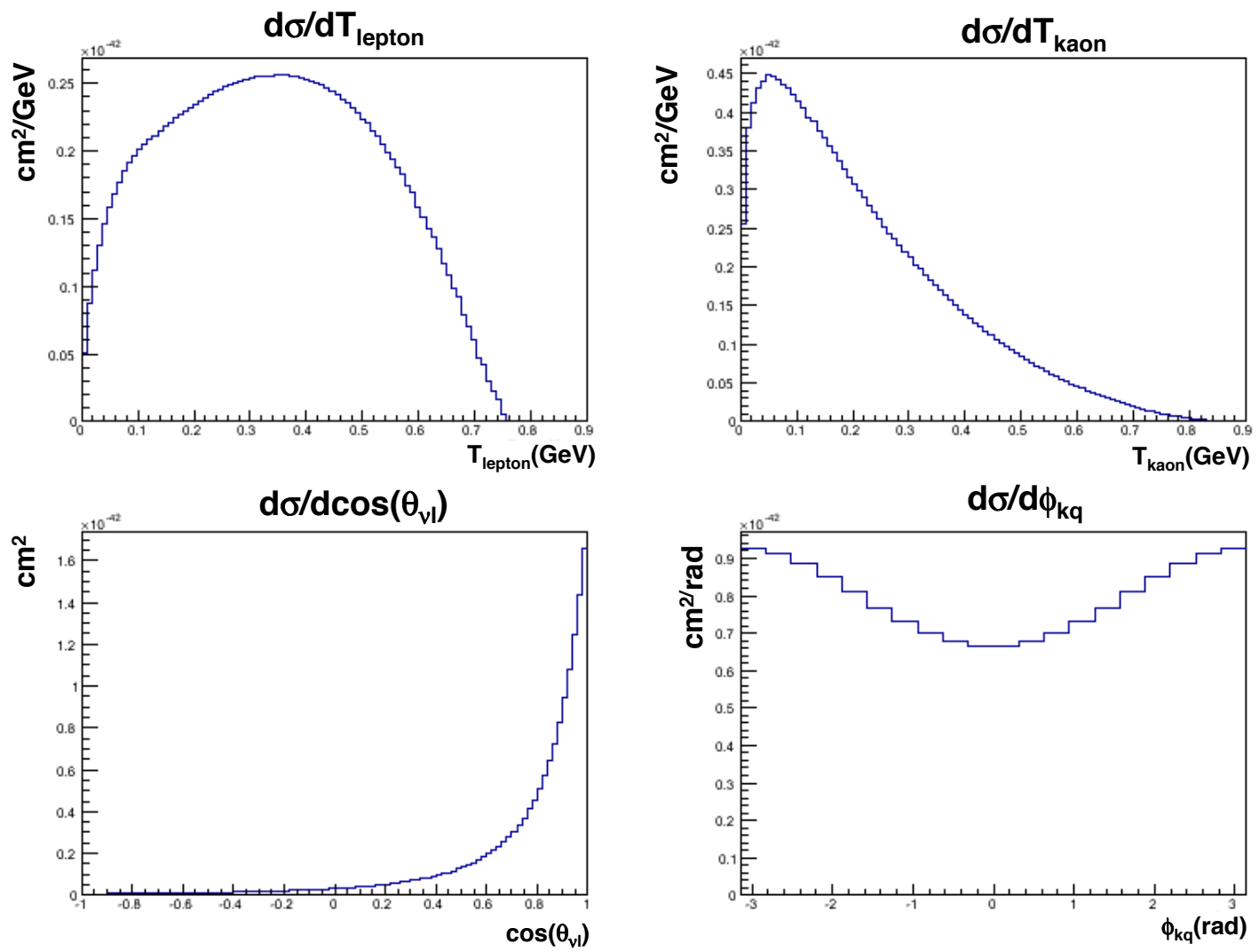

Figure 9: Kinematic distributions for the scattering of $1.5 \mathrm{GeV}$ neutrinos in the channel $\nu_{\mu}+p \rightarrow$ $\mu^{-}+K^{+}+p$. 


\section{Tools and Experimental Interfaces}

We made a number of improvements and fixes to tools and interfaces distributed with GENIE. In particular, for this release, we made changes to the flux drivers, the reweighting machinery, and to some of the event generation applications. Where possible, we provide code snippets to support the changes discussed in the text.

\subsection{Flux Drivers}

In order to provide better flexibility to users in applications that utilize flux drivers, three significant changes were made in this release:

1. A GFluxDriverFactory has been implemented; this allows flux drivers to self-register (Code1) with the factory and be returned from the factory by providing the name as a string (Code 2 ). This will allow more applications to use different drivers interchangably and for expansion of the available list by loading a library containing as self-registering driver without need to rebuild the application.

2. A common flux interface GFluxFileConfigI (Code 3) was introduced to unify configuration of flux drivers that depend on external file sets, such as ntuple-based drivers. These additions allow GNuMIFlux, GSimpleNtpFlux and the external GDk2NuFlux to be used completely interchangeably with any user configuration taking place via passed strings. It also unifies setting a limit on what neutrino flavors to return. For ROOT ntuple-based drivers it unifies an interface for getting the underlying branch objects, allowing users to copy the corresponding detailed flux records to accompany a generated event. Other flux drivers may start to incorporate these interfaces for additional interchangeability.

3. A second new common interface GFluxExposureI (Code 4) unifies flux drivers that can report an "exposure" such as time or protons-on-target. Initially only GNuMIFlux, GSimpleNtpFlux and the external GDk2NuFlux have been migrated to use this; future migration of others would expand the interchangablility.

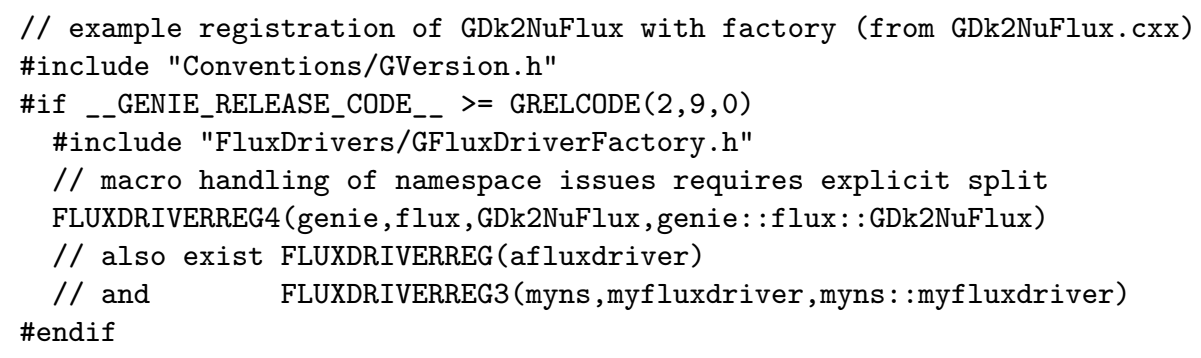

Code 1: Example code for registering new flux drivers with the factory 


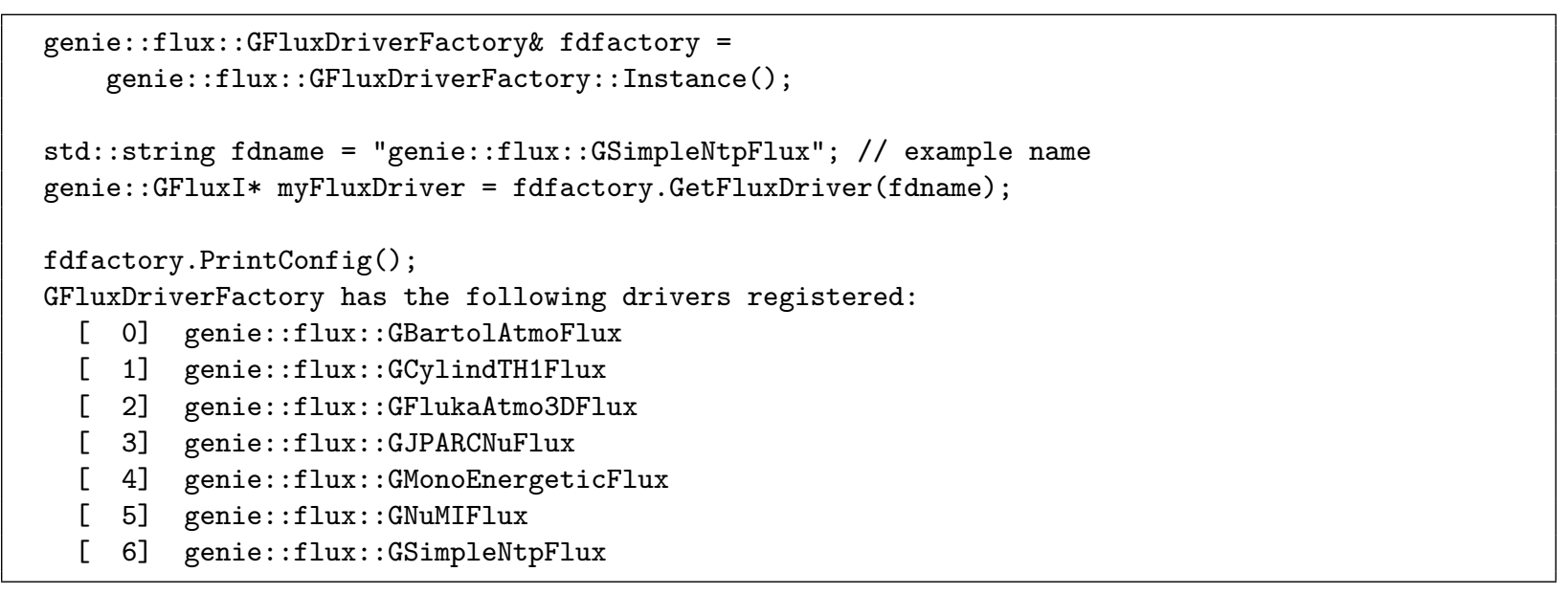

Code 2: Example code for GFluxDriverFactory

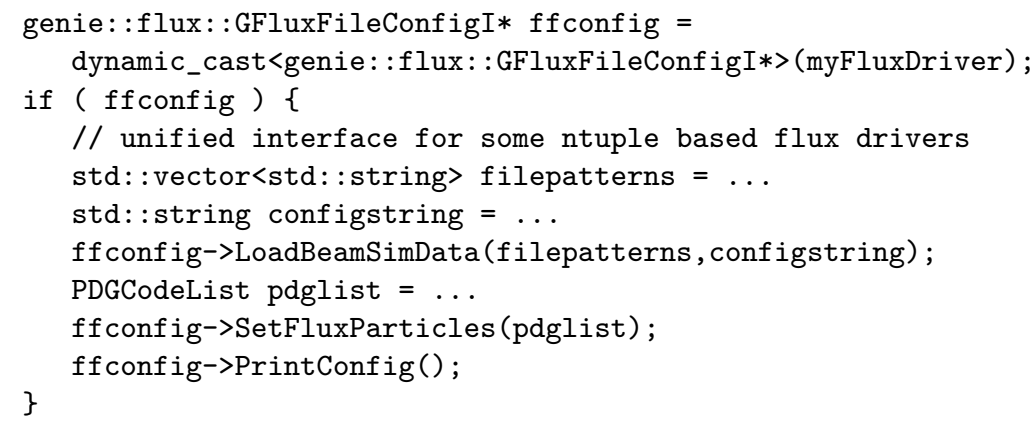

Code 3: Example code for GFluxFileConfigI interface

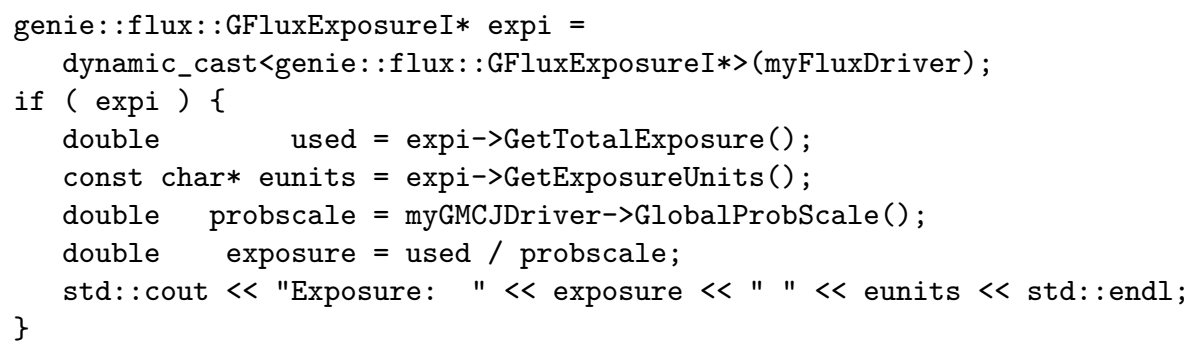

Code 4: Example code for GFluxExposureI interface 


\subsection{Reweighting}

Two adjustments were made to the reweighting machinery:

1. In GENIE Release 2.8.2, the treatment of formation zones was improved and introduced separate parameters for formation times for mesons and nucleons. The formation zone reweighing code has now been updated to reflect these changes.

2. Prior to this release, the kRDcyTwkDial_Theta_Delta2Npi knob in GENIE only affected the pion angular distribution for $\Delta(1232)^{++}$events. This has now been changed so that the reweighing is applied to all charge states of the $\Delta(1232)$.

\subsection{Event Generation Applications}

The gevgen_numi application was renamed to gevgen_fnal to indicate the wider applicability (NuMI, DUNE and booster based experiments). The executable will dynamically pick up the external GDk2NuFlux flux driver if available (i.e. there is no longer a build dependence of GENIE on $\mathrm{Dk} 2 \mathrm{Nu}$ for this feature). For all supported (ntuple-based) beam related flux drivers the flux entry used from the input will be copied to a branch along side the GHepRecord and metadata from all input files will be copied to the output file.

\section{$5 \quad$ Technical Updates}

We made a small set of changes to the core "technical" components of GENIE. We define technical changes as those modifying the framework, framework interfaces, the event record, the configuration system, and the build system.

For GENIE 2.10.0 we restored the build Makefile structure from GENIE 2.8.0. In GENIE 2.8.2 we introduced a change that would stop the build on any error. We have returned to the 2.8.0 behavior of continuing to attempt to build libraries even after one library has failed.

\subsection{Event Record Updates}

Three changes were made to the GENIE event record, enumerated below. Please note that this change is not backwards compatible in the sense that events produced with GENIE 2.10 will not be successfully read by older versions of GENIE. Because GENIE uses ROOT for persistency, it should be possible to read event produced by older versions of GENIE.

1. An additional value has been added to the enumeration in the ScatteringType class to identify single kaon events, as described above.

2. The $\mathrm{XClsTag}$ object has been updated to include information needed to tag $\Delta S=1$ events, which can now be accessed via the isStrangeEvent and StrangeHadronPdg methods.

3. Reintroduced the DiffXSecVars method which allows a query of the GHepRecord for the current KinePhaseSpace_t value. 


\section{Conclusions}

While the default physics in GENIE 2.10.0 is largely the same as the last production series, GENIE 2.8, this release has made some important technical changes and introduced a set of new models for advanced users.

\section{$7 \quad$ Acknowledgements}

We are grateful to many members of the neutrino physics community who provided feedback on development versions of this release. In particular, we would like to thank: I. Kagorin for comments on the KLN resonance model, R. Gran and J. Schwehr for a careful review of some numerical routines, T. Le for discovering a mistake in the Delta re-weighting code, and L. Alvarez-Ruso for finding a small mistake in the neutral current elastic scattering model.

This work was supported by the Fermi National Accelerator Laboratory, operated by Fermi Research Alliance, LLC under Contract No. De-AC02-07CH11359 with the United States Department of Energy. Work at Tufts University was supported by Department of Energy grant de-sc0007866 Work at the University of Pittsburgh was supported by Department of Energy grant de-sc0007914

\section{References}

[1] C. Andreopoulos et al., Nucl.Instrum.Meth. A614, 87 (2010), 0905.2517.

[2] T. Sjostrand, S. Mrenna, and P. Z. Skands, JHEP 0605, 026 (2006), hep-ph/0603175.

[3] R. Brun and F. Rademakers, Nucl.Instrum.Meth. A389, 81 (1997).

[4] M. Whalley, D. Bourilkov, and R. Group, (2005), hep-ph/0508110.

[5] A. Bodek, M. Christy, and B. Coopersmith, Eur.Phys.J. C74, 3091 (2014), 1405.0583.

[6] C. Llewellyn Smith, Phys.Rept. 3, 261 (1972).

[7] E. J. Moniz et al., Phys. Rev. Lett. 26, 445 (1971).

[8] A. A. Aguilar-Arevalo et al., Phys. Rev. D 81, 092005 (2010).

[9] A. A. Aguilar-Arevalo et al., Phys. Rev. D 88, 032001 (2013).

[10] L. Fields et al., Phys. Rev. Lett. 111, 022501 (2013).

[11] G. A. Fiorentini et al., Phys. Rev. Lett. 111, 022502 (2013).

[12] V. Lyubushkin et al., The European Physical Journal C 63, 355 (2009).

[13] T. W. Donnelly and I. Sick, Phys.Rev. C60, 065502 (1999), nucl-th/9905060.

[14] J. Carlson, J. Jourdan, R. Schiavilla, and I. Sick, Phys.Rev. C65, 024002 (2002), nuclth/0106047. 
[15] C. Maieron et al., Phys.Rev. C80, 035504 (2009), 0907.1841.

[16] M. Martini, M. Ericson, G. Chanfray, and J. Marteau, Phys.Rev. C80, 065501 (2009), 0910.2622 .

[17] J. E. Amaro, M. B. Barbaro, J. A. Caballero, T. W. Donnelly, and C. F. Williamson, Phys.Lett. B696, 151 (2011), 1010.1708.

[18] M. Martini, M. Ericson, G. Chanfray, and J. Marteau, Phys.Rev. C81, 045502 (2010), 1002.4538 .

[19] J. Nieves, I. R. Simo, and M. J. Vicente Vacas, Phys.Lett. B721, 90 (2013), 1302.0703.

[20] A. Bodek, H. Budd, and M. Christy, The European Physical Journal C 71 (2011).

[21] T. Leitner, O. Buss, L. Alvarez-Ruso, and U. Mosel, Phys. Rev. C 79, 034601 (2009).

[22] E. J. Moniz, Phys. Rev. 184, 1154 (1969).

[23] R. Smith and E. Moniz, Nuclear Physics B 43, 605 (1972).

[24] O. Benhar, S. Fantoni, G. I. Lykasov, and N. V. Slavin, Phys. Rev. C 55, 244 (1997).

[25] O. Benhar, S. Fantoni, and G. Lykasov, The European Physical Journal A - Hadrons and Nuclei 7, 415 (2000).

[26] J. E. Amaro et al., Phys. Rev. C 71, 015501 (2005).

[27] A. Bodek and J. L. Ritchie, Phys. Rev. D 23, 1070 (1981).

[28] A. Bodek and J. L. Ritchie, Phys. Rev. D 24, 1400 (1981).

[29] T. Yang, C. Andreopoulos, H. Gallagher, K. Hoffmann, and P. Kehayias, Eur.Phys.J. C63, 1 (2009), 0904.4043.

[30] SKAT, N. Agababyan et al., Phys.Atom.Nucl. 74, 229 (2011), 0811.2343.

[31] D. Ashery et al., Phys.Rev. C23, 2173 (1981).

[32] I. Navon et al., Phys.Rev. C28, 2548 (1983).

[33] T. Bowles et al., Phys.Rev. C23, 439 (1981).

[34] LADS, A. Lehmann et al., Phys.Rev. C60, 024603 (1999).

[35] K. Aniol et al., Phys.Rev. C33, 208 (1986).

[36] B. Brinkmoeller and H. Schlaile, Phys.Rev. C48, 1973 (1993).

[37] A. Clough et al., Nucl.Phys. B76, 15 (1974).

[38] S. Mashnik, R. Peterson, A. Sierk, and M. Braunstein, Phys.Rev. C61, 034601 (2000). 
[39] S. Mashnik, A. Sierk, K. Gudima, and M. Baznat, J.Phys.Conf.Ser. 41, 340 (2006), nuclth/0510070.

[40] A. Saunders et al., Phys.Rev. C53, 1745 (1996).

[41] E. Friedman et al., Phys.Lett. B257, 17 (1991).

[42] K. Nakai et al., Phys.Rev.Lett. 44, 1446 (1980).

[43] GNU Scientific Library, http://www.gnu.org/software/gsl/, 2015.

[44] C. Berger and L. Sehgal, Phys.Rev. D76, 113004 (2007), 0709.4378.

[45] K. S. Kuzmin, V. V. Lyubushkin, and V. A. Naumov, Mod.Phys.Lett. A19, 2815 (2004), hep-ph/0312107.

[46] D. Rein and L. M. Sehgal, Annals Phys. 133, 79 (1981).

[47] W. Metcalf and J. Nowak, Understanding the $Q^{2}$ discrepancy in the $\mathrm{CC} \pi^{+}$sample, BooNE Technical Note 260, 2009.

[48] MiniBooNE, J. A. Nowak, AIP Conf. Proc. 1189, 243 (2009), 0909.3659.

[49] M. Rafi Alam, I. Ruiz Simo, M. Sajjad Athar, and M. Vicente Vacas, Phys.Rev. D82, 033001 (2010), 1004.5484. 